\title{
Romain Rolland et Elsa Nüesch
}

La rencontre avec une jeune Suissesse perspicace et militante :

Correspondance (1916-1931)

Jean-Pierre Meylan

\section{(2) OpenEdition}

\section{Journals}

Édition électronique

URL : http://journals.openedition.org/edl/350

DOI : $10.4000 /$ edl. 350

ISSN : 2296-5084

Éditeur

Université de Lausanne

\section{Édition imprimée}

Date de publication : 15 septembre 2012

Pagination : 173-212

ISBN : 978-2-940331-28-4

ISSN : 0014-2026

Référence électronique

Jean-Pierre Meylan, «Romain Rolland et Elsa Nüesch », Études de lettres [En ligne], 3 | 2012, mis en ligne le 15 septembre 2015, consulté le 22 décembre 2020. URL : http://journals.openedition.org/edl/ 350 ; DOI : https://doi.org/10.4000/edl.350 


\section{ROMAIN ROLLAND ET ELSA NÜESCH LA RENCONTRE AVEC UNE JEUNE SUISSESSE PERSPICACE ET MILITANTE: CORRESPONDANCE (1916-1931) ${ }^{1}$}

La rencontre de Romain Rolland (50 ans) avec une jeune étudiante de Saint-Gall (22 ans) qui en rend compte et qui devient plus qu'une admiratrice: une intellectuelle, une enseignante et une pionnière des droits de la femme. Non seulement en Suisse romande, mais aussi en Suisse alémanique, Rolland - à l'exemple de Tolstoï avant 1914 - était entouré d'une phalange internationale de jeunes adeptes pacifistes qui firent parler d'eux par la suite dans l'entre-deux-guerres, tels Charles Baudouin, Ivan Goll, Henri Guilbeaux, Pierre Jean Jouve, Edmond Privat, Frans Masereel, Claude LeMaguet, Gaston Thiesson ${ }^{2}$, entre autres. A ce groupe s'ajoute Elsa Nüesch, une des rares femmes parmi eux, qu'il convient maintenant de mieux faire connaître.

\section{Elsa Nüesch (1894-1952)}

Elsa Nüesch est née le 13 novembre 1894 à Saint-Gall. Elle y meurt en 1952. Sa famille comprend son père, Jakob Nüesch, caissier régional des CFF, sa mère, Marie Nüesch-Keller, couturière de formation et sa sœur Alice, institutrice. La sœur meurt prématurément en 1928, la mère en 1934.

I. Remerciements à M. le professeur Bernard Duchatelet, pour son expertise et son soutien, à Mme Colette Girard (Fribourg), pour la transcription de quelques lettres, et à Mmes Martha Gosteli, Monika Bill et Regula Schär du Gosteli-Archiv de Worblaufen dans le canton de Berne.

2. Pour ne pas oublier les Georges Duhamel, Charles Vildrac, Jean-Richard Bloch, Marcel Martinet, Jean de Saint-Prix et autres, de la même famille de pensée, mais qui ne purent ou ne voulurent pas s'expatrier à Genève pour toute la durée de la guerre, tout en restant en communion avec Rolland en Suisse. 
Elsa se décrit, dans une sorte de "curriculum-confession " rédigé peu avant sa mort, comme éprise de poésie, timide, légèrement handicapée par une santé frêle à la suite d'une grave opération en 1909, peu à l'aise dans sa scolarité. Elle en aurait été conditionnée au point de se sentir isolée, peu sociable et repliée sur son univers intérieur pendant toute sa vie. Son école du Thalhof à Saint-Gall voulant se doter d'une section de lycée (Gymnasium) et n'y réussissant pas, elle fut mutée à la section "commerciale». Mais elle réussit à rattraper le latin et les mathématiques nécessaires et put entrer au Gymnasium, puis à l'Université de Neuchâtel, bénéficiant ainsi d'une éducation culturelle alors réservée à une partie infime des jeunes filles de son époque, surtout en Suisse orientale. Bien qu'on la jugeât inapte à l'enseignement, elle deviendra par la suite la première enseignante à formation académique à occuper un poste permanent dans un lycée suisse.

Elle commença à publier des poèmes et articles de circonstance dans la Schweizerische Frauenzeitung. Dès lors, encore étudiante, elle ne cessa de livrer des articles aux journaux et devint une journaliste régulière du quotidien St.Galler Tagblatt à partir de 1930 (souvent en se cachant derrière les sigles E.N. ou * pour ne pas être perçue comme femme).

A Neuchâtel, où elle fit ses études, elle apprit le français et soutint sa thèse sur Nietzsche et l'antiquité (1925, publiée aux PUF) sous la direction de Carl Albrecht Bernoulli ${ }^{3}$. Ce fut son seul point d'attache avec la francophonie, hormis une période où elle enseigna dans un collège privé à Bex (VD), car les aléas de sa biographie la privèrent d'un contact plus direct avec la France. Elle fit également des études à Zurich et à Munich pendant la Première Guerre mondiale - circonstance qui la mit en relation avec Romain Rolland. En politique, elle ne fut pas moins hardie: elle participa à la section féminine du parti socialiste du canton de Saint-Gall et y milita pour les droits égaux des femmes en Suisse orientale, région particulièrement rétive à ce sujet, la Suisse étant le dernier pays en Europe où l'on généralisa le vote des femmes. En 1930, elle fut nommée professeur de français et de latin au lycée (Gymnasium) de la Kantonsschule de Saint-Gall (lycée cantonal), poste qu'elle occupa

3. Carl Albert Bernoulli (1868-1937): théologien protestant et philosophe bâlois, collaborateur du Nietzsche-Archiv à Bâle et auteur de Friedrich Nietzsche und Franz Overbeck (1908). 
jusqu'en 1950 lorsqu'elle dut y renoncer à cause de la maladie qui la rongeait.

Saint-Gall et la Suisse orientale, assez prospères avant 1914, dépendaient de l'industrie du textile et de la broderie - occupations fortement cycliques. Cette prospérité s'effondra dès 1917 où les villes connurent la faim et le chômage comme en Allemagne et furent subséquemment ravagées par la seconde crise, celle des années 30 . Une situation très peu favorable pour le développement professionnel d'une jeune femme qui voulait faire son chemin dans la vie et qui était en outre handicapée par sa condition et sa profession de foi politique minoritaire. Dans cette situation, vouloir embrasser un métier et mener une vie familiale était quasiment impensable, surtout avec une formation universitaire, car partager le temps entre la vie et l'usine était la condition des femmes ouvrières faiblement rémunérées ${ }^{4}$.

En 1932, elle plaida dans une revue politique importante la cause de la compatibilité des carrières féminines avec la vie familiale ${ }^{5}$, une position qui n'était même pas partagée par les socialistes et les milieux ouvriers classiques où l'on pensait qu'en temps de chômage, les femmes occupant une profession constituaient une concurrence déloyale aux hommes et sapaient les foyers.

Dès 1936, en pleine crise économique, tout en continuant à se situer à la gauche démocratique, elle fut prise par la fièvre patriotique qui se développa à la veille de la Seconde Guerre mondiale, à une époque dite de la geistige Landesverteidigung, "défense nationale intellectuelle», qui se caractérisait par le retour à la neutralité intégrale de la Suisse et par la lutte contre les fascismes. Elle ne cessa de lutter comme Rolland contre le fascisme, notamment contre l'infiltration en Suisse d'une pensée autoritaire venant d'Italie et d'Allemagne.

A cette époque, toutefois, l'appartenance d'Elsa Nüesch à la socialdémocratie lui conféra une légitimité suffisante pour occuper un poste d'Etat dans un lycée du canton de Saint-Gall, ce parti étant considéré comme suffisamment staatstragend ("loyal envers l'Etat»). En revanche, afficher des sympathies pour Romain Rolland dans les années 30

4. Seules les (rares) institutrices pouvaient alors cumuler mariage et profession; jusque dans les années 60 , ceci était incompatible pour les enseignantes de lycées.

5. Die weibliche Berufsarbeit auf der Suche nach ihren eigenen Zielen, dans Politische Rundschau, Bienne, année 11 (1932), c. 7, p. 238-246. 
- comme ce fut le cas encore en 1926 lors de son $60^{\mathrm{e}}$ anniversaire - était de plus en plus mal vu, car celui-ci avait basculé de l'antifascisme largement partagé par les avant-gardes vers un philo-soviétisme ouvertement professé - ce qui n'était pas du goût de l'entourage d'Elsa Nüesch, ni de ses admirateurs suisses ${ }^{6}$. En 1936, l'année de son $70^{\mathrm{e}}$ anniversaire, il n'y eut plus que les gauches pour fêter Rolland. Plusieurs cantons et finalement la Confédération passèrent des ordonnances qui interdisaient aux fonctionnaires d'appartenir au parti communisme, sous peine d'exclusion, et l'importation de beaucoup de publications communistes fut interdite, dont L'Humanité.

Ce revirement était représentatif de l'impact de Rolland dans les pays de langue allemande: énormément lu entre 1920 et 1934, sa fortune baissa dès lors et on ne voulut plus que se souvenir de l'auteur du JeanChristophe, soit d'un auteur irénique et conciliant. Le cas de Nüesch présage le clivage dans l'impact posthume de Rolland: plus il était canonisé comme saint du pacifisme sous les régimes socialistes, plus il passa au purgatoire et fut ostracisé à l'ouest.

Après une première série de cours à l'Université populaire de Neuchâtel dans les années 20 (institution alors très à l'avant-garde des idées politiques et sociales), ce fut dans les années 30 qu'Elsa Nüesch commença régulièrement à donner des cours à la Handelshochschule de Saint-Gall, qui allait devenir l'Université de Saint-Gall dans les années 80. Parmi ses cours littéraires (sur Verhaeren, Bergson, Nietzsche, entre autres) ne manque pas, bien sûr, un cours sur Romain Rolland dont

6. Pour clarifier toute équivoque sur le «communisme» de Rolland: Bernard Duchatelet, dans ses diverses études sur le Rolland politique, a montré que Rolland (1) était un "compagnon de route» du communisme et "philo-soviétique» en ce sens qu'il croyait au renouveau de la Russie par la Révolution, (2) ne s'était parfois laissé «embrigader» que contre son gré et à son insu, (3) qu'il n’avait jamais formellement appartenu au PCF, dont il n'a jamais accepté la discipline de parti. Ce qui n’a jamais empêché le PCF de le proclamer un des siens quand cela l'arrangeait. Voir B. Duchatelet, Voyage à Moscou. Si Elsa Nüesch avait été affiliée au Parti Communiste Suisse (interdit en 1939), elle n'aurait plus pu exercer sa profession d'enseignante, fonctionnaire d'Etat. L'éditeur suisse des traductions allemandes de Rolland, Emil Roniger, eut le courage de braver l'interdiction nazie de publication des œuvres "politiques" de Rolland en Allemagne, en 1934, mais ne suivit pas Rolland dans son «implication" dans le régime soviétique à partir de 1935, donc de son voyage à Moscou. On peut dater d'environ 1936 le moment où Rolland prit la décision de quitter la Suisse, ce qu'il fit en 1938. En Suisse, il ne se sentait plus bienvenu et le Front populaire en France stimulait tous les espoirs. 
nous citerons ici quelques extraits et pour lequel elle put puiser dans son expérience personnelle.

Pendant la Seconde Guerre mondiale, Elsa Nüesch participa à la mise en place du Service auxiliaire féminin de l'armée en 1940 (fondé à l'origine par des associations féminines, avant d'être récupéré par un corps d'officiers à l'habitus autoritaire), s'impliqua dans les services sociaux de l'armée, qui attiraient les élites parmi les femmes, et conduisit un servicebibliothèque pour soldats.

Les papiers d'Elsa Nüesch sont conservés par chance dans les Archives Gosteli à Worblaufen, près de Berne (qui se consacrent à la mémoire de la lutte des droits des femmes). Elsa Nüesch est bien connue dans ce domaine, mais non comme correspondante de Rolland, un souvenir escamoté par l'anticommunisme dominant en Suisse orientale après $1945^{7}$.

\section{Les circonstances d'une rencontre insolite}

En abordant cette correspondance atypique pour Rolland, il faut s'imaginer la situation suivante: une étudiante de 22 ans, dont la langue française n'est pas la langue maternelle, débutante dans cette langue, étudiante en lettres, candide mais déterminée, revenant d'un séjour d'études à Munich, en 1916, en pleine guerre, s'adresse à l'auteur de Jean-Christophe et surtout à celui d' «Au-dessus de la mêlée», alors âgé de 50 ans et très célèbre après s'être vu attribuer le Prix Nobel la même année. Rolland était déjà submergé de lettres d'admiratrices et sollicité de toutes parts; donc peu de chance d'attirer son attention. Néanmoins, il fut subjugué par la qualité de jugement et d'observation d'Elsa Nüesch, au point qu'il copia des pages entières des lettres d'Elsa dans son Journal des Années de Guerre 1914-1919 (cité par la suite $J A G$ ). Le JAG fut publié en 1952, l'année de la mort d'Elsa; le lien entre elle et Rolland passa inaperçu.

7. En 1945, le peintre suisse Hans Erni, actif au sein de l'Association Suisse-Union Soviétique, honora Rolland de manière posthume par un portrait dont l'existence fut escamotée au public jusque dans les années de dégel. Les commanditaires publics préféraient ses fresques épico-patriotiques d'avant-guerre. 
Grâce au Gosteli Archiv et à trois lettres conservées à la Bibliothèque nationale de France $(\mathrm{BnF})$, nous disposons maintenant des deux volets: les lettres d'Elsa recopiées en partie par Rolland (dans $J A G$ ) et ses réponses (sous forme de copies de textes olographes). En plus, nous disposons des extraits des manuscrits de cours d'Elsa Nüesch (textes inédits, relatant la rencontre avec Rolland).

La période des échanges les plus intensifs se situe dans les années 19161917, années pivots de la guerre, quand les pacifistes des deux camps cherchaient une issue "honorable" (débat autour des propositions du président Wilson), quand le débat intérieur en France et en Allemagne était encore relativement ouvert - par rapport à l'année 1918, envenimée par la lutte contre le défaitisme et l'idéologie du combat acharné pour la "dernière des guerres», la guerre sous-marine, l'arrivée des Américains et la révolution russe. La correspondance s'espace dès lors dans les années 20 où Elsa Nüesch tente de reprendre le fil sans que Rolland n'y mette de l'entrain. Dans les deux dernières lettres conservées ( $n^{\circ} 12$ du 24 janvier 1926 et $\mathrm{n}^{\circ} 13$ du 15 avril 1931), elle résume les étapes parcourues, fait état de ses difficultés comme enseignante et indique combien Rolland est tombé en disgrâce politique dans son entourage en Suisse orientale. Se targuer d'avoir fait connaissance de Rolland y était, de plus en plus, un handicap dans la carrière de cette enseignante de lycée et quasiment maître de conférences à la future Université de Saint-Gall.

La correspondance se termine en avril 1931, après une tentative spontanée d'Elsa de sonner à la porte de la Villa Olga à Villeneuve (où elle est éconduite, Rolland se disant malade), suivie par des excuses et des explications d'Elsa sur un ton légèrement plaintif (lettre du 15 avril 1931) et le billet - quasiment d'adieu - de Rolland du 24 avril 1931.

Bilan de cette rencontre: une nouvelle fenêtre s'ouvre pour illustrer la fortune et l'impact de Romain Rolland en Suisse - témoignage d'une rafraîchissante candeur, mais aussi de courage et de grande perspicacité. Cette rencontre correspond à une constante chez Rolland: son habitude de s'adresser aux jeunes générations auxquelles il aimait lancer des appels, affectant le rôle d'un "gourou» comme le fit jadis Tolstoï. Rappelons que plus tard, une autre étrangère, russe celle-là aussi, elle aussi bien plus jeune que Rolland et de la génération d'Elsa, réussit à capter son attention: Maria Pavlovna Koudacheva (1895-1985), qui devint son épouse en 1934. C'est aussi une pièce à verser à l'histoire du succès énorme de Romain Rolland dans les pays germanophones (avant la proscription en 
Allemagne, en 1934), qui fit de lui un grand sage pacifiste et intégrateur des peuples, alors qu'il passait pour un partisan et un militant en France même.

Jean-Pierre Meylan

Chercheur indépendant, Bâle 


\section{CORRESPONDANCE ROMAIN ROLLAND - ELSA NÜESCH}

(1916-1931)

\section{$N^{\circ} 1$ : Elsa Nüesch à Romain Rolland, 20 janvier $1916^{8}$}

Neuchâtel, le 20 janvier 1916

\section{Monsieur,}

Pardonnez-moi, Monsieur, d'avoir remis à si tard, la réponse à votre très aimable lettre. J'en suis bien honteuse je ne saurai m'excuser.

Elle m'a causé une joie profonde et elle m'inspirait bien de [la] reconnaissance envers vous, Monsieur, qui au milieu de vos occupations vous êtes souvenu de témoignage d'affection envoyé de ma part. L'impression a été trop forte et je n'osais vous en remercier tout de suite.

Alors, j'attendis mes vacances de Noël que je passais dans ma ville natale et ma joie était grande de voir toutes les sympathies aller vers vous, Monsieur. Bien sûr, il y a encore parmi mes concitoyens des francophobes irréductibles mais ceux, qui sont formés à l'esprit français, sont en grande minorité. Tous, parmi nos gens qui sont à peu près instruits, ont parlé de vous avec enthousiasme. Vous connaissez, Monsieur, mieux que moi, l'enthousiasme neuf de notre race, il n'est pas éteint. Dans les pays comme le nôtre où la haine n'a pas rendu aveugle les consciences,

8. Les lettres antécédentes ne sont pas conservées. Les lettres 1, 12 et 13 proviennent du fonds Romain Rolland de la BnF. Elsa était alors encore en train d'apprendre le français à Neuchâtel, ce qui explique les maladresses, ses phrases tortueuses calquées sur l'allemand. La lettre du 20 janvier 1916 est encore assez confuse. Rolland, en copiant les autres lettres d'Elsa dans son $J A G$ les a corrigées lui-même, car on n'y trouve pas les maladresses habituelles (il semble qu'il l'ait aussi fait avec d'autres correspondants allophones, dont Stefan Zweig). Le français d'Elsa s'améliore au courant de la correspondance, mais n'atteint jamais la fluidité. Dans cette transcription nous nous rapprochons du parti pris de Rolland et éliminons toutes les bévues ou fautes évidentes et explicitons entre [ ] des tournures incompréhensibles, souvent empruntées à l'allemand. 
on croit encore à toute grande et bonne pensée, à tout acte libérateur d'où il vient. On se réjouit d'autant plus quand une telle idée vient de la France. La France a toujours eu son prestige et c'est de la France que nous attendons à tort ou à raison qu'elle se délivre de la haine la première. Qu'elle délivre le monde de la haine, qu'elle soit le grand foyer des intelligences. On a cherché avec inquiétude, si parmi les belligérants se trouvaient quelques êtres libres et forts qui fussent au-dessus de la mêlée. On connaît un peu - un peu seulement - votre grande œuvre, mais l'intérêt y est, il s'agrandit de jour en jour. Ce n'est pas en vain que Monsieur Paul Seippel ${ }^{9}$ a fait ses conférences dans notre pays - ses paroles ont laissé des empreintes profondes. Même [chez] les plus récalcitrants ont [il a] laissé des empreintes profondes. Même les plus récalcitrants veulent entendre et lentement mais d'un progrès sûr, votre pensée [qui] pénètre les intelligences et les âmes. Ce ne sont plus les intellectuels seuls, heureusement c'est le peuple qui de plus en plus se laisse pénétrer par les paroles surprenantes que vous prononcez. On associe votre nom au [Grand Meaulnes] ${ }^{10}$ et on n'a pas tort, je vois, puisqu'il y a une si profonde parenté d'esprit et d'âme entre [ces] œuvres.

Pourquoi vous dire tout cela? Je sais bien que vous ne tenez point à entendre des paroles flatteuses, et un enthousiasme juvénile et irraisonné ne peut non plus vous être de grande valeur. Mais je pense espérer que la sympathie d'un peuple qui est un lien entre les autres, ne vous est pas indifférente. Je me figure que cela peut même vous montrer combien votre grande œuvre est [déjà] nécessaire, indispensable à l'heure. Et peutêtre cela vous montre un peu le progrès de votre œuvre.

C'est peut-être un peu un honneur pour notre pays, qu'il a eu des idées semblables aux vôtres, Monsieur Romain Rolland. On vous invite bien moins qu'on cherche [plutôt] à collaborer. Tout naturellement on se sent [en] sympathie avec vos pensées, avec vos grandes visions, avec JeanChristophe «tout le ciel qui appartient à l'aigle». On voit comme[nt] vous avez bien compris la Suisse, si mal comprise aujourd'hui. Vous nous

9. Paul Seippel (1858-1926) fut rédacteur du Journal de Genève, romancier et détenteur de la chaire de littérature française et romande à l'Ecole Polytechnique de Zurich. Il est l'auteur du premier essai biographique sur Romain Rolland, Romain Rolland, l'homme et l'œuvre, Paris, Ollendorf, Genève, Payot, 1913.

Io. Nom peu lisible. Il est probable qu'elle parle du Grand Meaulnes d'Alain-Fournier (1913), car cette œuvre faisait partie des œuvres incontournables utilisées à l'époque dans l'enseignement du français en Suisse et Elsa Nüesch apprenait alors le français. 
faites un bien grand honneur de nous juger si bien. Mais votre jugement surtout m'importe beaucoup parce que vous n'êtes point optimiste, point enthousiaste. Vous devez bien connaitre nos défauts et nos peines, nos luttes intérieures et les malencontreuses méprises qui se trouvent partout. Nous ne sommes point meilleurs que les autres, on a bien raison de se le répéter. Mais ce n'est pas en vain non plus que C. Spitteler ${ }^{11}$ a parlé. Il nous a montré d'une manière frappante, presque rude, nos défaillances, vous avez dit dans votre lettre que vous admirez le génie de C. Spitteler, vous dites que votre peuple ne saurait assez l'aimer.

Et voilà ce qui est presque triste. Carl Spitteler ne compte pas beaucoup d'amis dans notre pays. C'est avec peine presque qu'on se met à le lire. Quelle peine n'ai-je eue pour assurer mes bonnes connaissances, qu'on peut lire Le Printemps olympien, qu'on peut en jouir, que c'est un poème qui est très suisse et universel. On s'attache aux petites choses, on n'est pas habitué aux hardiesses, aux traits de génie qui distinguent l'œuvre de C. Spitteler. Et puis il a le malheur presque d'être un des nôtres. G. Keller, C. F. Meyer et Spitteler ont toujours eu de la peine à pénétrer. Le trait est bien suisse et bien germanique; aussi on lira plus facilement telle œuvre française que telle de Spitteler. Il est le Prométhée des nouvelles générations qui n'aiment plus travailler, tournoient craintivement dans le cercle tracé par nos pères. Un dennoch ${ }^{12}$ surgit dans bien des consciences. On sent qu'il y aura mieux à faire que les petites agaceries locales et les guérillas des partis. On sent qu'il faut élargir l'horizon. La grande lutte nous impose des vues plus grandes. Nous ne pouvons pas faire des actes d'héroïsme comme ceux qui vont à la mort pour leur patrie. Nous ne pouvons partager l'enthousiasme fiévreux d'aucun d'eux. Mais il y a autre [chose] à faire. On peut travailler pour la paix. On peut veiller sur tout. On peut offrir refuge aux pensées proscrites comme les malheureux qui sont sans foyer. On peut donner accès à tous, les honorer et aimer les grandes âmes et les grandes œuvres de tous les pays. Si la

II. Rolland tint Carl Spitteler (1845-1924, auteur de poèmes épiques antiquisants) en très haute estime. Il y vit un allié dans sa lutte contre la haine fomentée entre alémaniques et romands. Elsa fait allusion au discours Notre point de vue suisse/Unser Schweizer Standpunkt prononcé en septembre 1914 devant la Nouvelle Société Helvétique, dans lequel il fait appel à l'unité nationale sans discrimination. Discours qui fit école et renforça l'esprit de la neutralité suisse.

I2. "malgré tout» ou «quand même». 
Suisse a donné quelque chose à Jean-Christophe, elle lui doit beaucoup à son tour. Elle dit qu'il est sien un peu et elle en sera fière.

Pardonnez-moi, Monsieur, si je vous adresse une prière, voudriezvous me faire savoir un jour si peut-être quelque paysage autour de ma ville natale a pu inspirer certaines pages de l'Adolescent. Ma question est presque ridicule, mais c'est d'un si vif intérêt. Je pense surtout à la fin de l'Adolescent, à la belle scène où il descend de la colline et revient vers la ville, après avoir laissé l'oncle Gottfried. Tout le passage, la scène me rappelle les collines qui entourent St Gall. C'est un détail, je le sais bien. Mais aussi saurais-je me représenter combien de visions, d'impressions fugitives peuvent aboutir à une œuvre d'art.

Peut-être que c'est dû à la grande connaissance des êtres que vous montrez dans Jean-Christophe qu'il y a surtout dans Le Buisson ardent et dans les premiers livres des personnages qui me semblent tous familiers, surtout Sabine, et puis surtout on est en Suisse.

Malheureusement, je me perds dans les détails et j'oublie que je n'ai voulu que [vous] remercier de toute mon âme dans cette lettre. En même temps, je sens que j'ai beaucoup de choses à vous dire et quand je les prononce, je suis honteuse de les voir si petites, si insignifiantes. Elles reviennent toutes au même sentiment, au sentiment de gratitude envers un seul homme et un sentiment douloureux d'être si insignifiant, chez ce sentiment aussi qui a tant fait pour retarder ma lettre, on recule devant les grandes joies, devant les grands témoignages; aussi pardonnez-moi encore une fois mon long silence, c'était l'émotion trop grande et l'impression trop vive.

Recevez l'assurance de ma profonde reconnaissance et de ma grande estime.

Elsa Nuesch

No 2: Elsa Nüesch à Romain Rolland, 11 novembre et 20 décembre 1916

$J A G$, p. 1023-1027:

D'une jeune étudiante à Neuchâtel, dont l'intelligence m'avait déjà frappé, Elsa Nüesch de Saint-Gall, un tableau saisissant de l'Allemagne transformée par la guerre (11 nov.-20 décembre):

... J'ai passé l'été de cette année en Allemagne, étudiant à l'Université de Munich, et je faisais de fréquents voyages dans le pays environnant. J'ai eu la 
chance d'entrer en contact avec toutes les classes de la société, de tous les partis et de toutes croyances. Et l'impression que cette Allemagne de 1916 a faite sur un étranger qui la visitait sans préjugé favorable ou haineux a été surprenante, inoubliable, triste. Le premier trait qui m'a frappée était la défaite de l'orgueil. L'orgueil que je craignais, qui d'avance me répugnait, n'était bien évident, bien "nourri", que chez les vieux bonshommes, les administrateurs, les écrivailleurs et un groupe d'officiers actifs, qui sont la lèpre du pays. Mais comme j'ai eu l'occasion de parler à des militaires de tous les grades, jusqu'au Ministre de la Guerre, j'ai été surprise de trouver chez tous ceux qui revenaient du front un état d'esprit comme personne ne s'en doute dans les autres pays. Je n'ai pas sous la main Au-dessus de la mêlée et l'article que vous avez consacré à la Littérature de guerre en Allemagne. Ce que j’ai vu et compris pendant mon séjour à Munich a confirmé pour moi la force de l'intuition de votre pensée. Il est vrai que la nouvelle génération d'Allemagne a pris conscience d'elle-même et s'oriente vers un autre but. Point de haine chez ceux qui reviennent du front; ils ne comprennent pas les gens qui peuvent encore émietter [sic] l'énergie pour haïr. Chez beaucoup d'entre eux, gens simples ou "gebildet" [cultivé], il y a un esprit de résistance contre ceux qui les ont lancés contre la France. "La France, que nous a-t-elle fait de mal? Nous ne savons pas. C'est insensé. Nous ne voulons ni ne pouvons plus les tuer. Pourquoi ceux qui avant la guerre prétendaient connaître la France, pourquoi n'ont-ils pas voulu voir? Malgré tous les chemins de fer, on se connaît moins que jamais. C'est une honte! Ce que nous avons vu nous restera imprégné dans le souvenir pour jamais. Nous n'aurions pas demandé mieux que de vivre dans notre "Frankenland". S'ils étaient venus chez nous, comme les Russes en Prusse Orientale, cela aurait été autre chose. Mais ainsi, on se fait haïr, sans savoir pour quel crime personnel. Mais la Belgique, voilà la plaie! Où vous hä̈ssent-ils le plus? Partout, mais surtout au nord, depuis Charleroi. Et c'est juste, malheureusement. Les premières armées y ont fait des ravages si atroces que nous devons nous en ressentir encore en 2000 (Wir werden's noch hundert Jahre nachher fühlen) ${ }^{13}$. Et nous l'aurions pu éviter, et cela aurait été si aisé! Quels idiots, nos officiers supérieurs! - Mais vous tous, vous avez toujours obéi? - Non. Si nos soldats avaient tout fait ce que les supérieurs commandaient, ils auraient commis infiniment plus de dégâts et de tort à ce pays. Les particuliers tâchent de réparer un peu le tort des gouvernants. Et plus tard il sera nécessaire à nous

13. Trad.: «Nous le ressentirons encore après cent ans.» 
tous de réparer, si on le peut encore. Vous le pourrez toujours, vous le pourrez, répugnés par l'exemple de vos supérieurs, vivre, travailler pour la paix et montrer aux autres peuples que vous du moins vous êtes dégoûtés de cette politique. - Mais les bons d'entre nous meurent dans la mêlée. "Il est vrai qu'à Munich du moins, le gouvernement doit tenir compte du peuple plus qu'ailleurs. Seule la guerre en dehors du pays a su empêcher une insurrection générale - non - une révolution. La révolte du pain du 23-24 juin 1916 n'a été qu'un avant-coureur. Spectacle émouvant, triste, fou et cruellement comique. Les soldats n'ont pas voulu tirer, et le peuple est resté vainqueur. J'ai été prise contre mon gré au milieu de la fournaise; et les gens, même harassés de travail, de fatigue et de faim, gardent leur rancune pour le moment de la paix. "On nous a mis des armes en mains... C'est nous qui avons sauvé la patrie, qui avons accompli tout le travail. On ne l'oubliera pas..." C'était comme un souffle précurseur d'un combat futur. Il y aura un Rütli, - peut-être un Tell ${ }^{14}$, qui, audacieux, brisera l'ennemi intérieur: Deine Uhr ist abgelaufen ${ }^{15}$.

(Ici, ma correspondante s'interrompt, un mois: Une maladie m'a forcée d'interrompre la lettre commencée. Je continue... : (20 décembre): ... Longtemps je n'ai pas voulu croire à ce revirement intérieur en Allemagne. Mais il a bien fallu... A voir, à questionner beaucoup de bonnes gens, j'ai reconnu qu'à Munich ils regardent derrière eux les premiers mois de la guerre avec un ébahissement ironique et douloureux. "Par où nous ont-ils fait passer? Par quel enfer! Hä̈r, toujours hä̈r! Un Bavarois ne se bat point pour un empereur élégant qui se galonne des travaux des autres... - Cela ne sert à rien! Vous vous êtes liés, soumis à la Prusse. Malgré votre bon fonds de Bavarois, vous êtes Prussiens malgré vous. Vous êtes cruelle; mais, en partie, vous avez raison ... Seulement, voyez, vous vivez chez nous bien plus librement que là-bas (en Prusse). Nous ne serons jamais Prussiens dans l'âme et cette guerre doit nous donner les moyens de nous rendre plus indépendants encore. Notre roi est autre; il vit pour nous, pour notre pays, et il se mêle au peuple. A Munich, les palais dans toute leur richesse sont simples, et les maisons des petits artisans sont pittoresques. Et dans notre vie à

I4. Lieu mythique au bord du lac des Quatre Cantons où les trois cantons primitifs de la Suisse auraient, selon, le Guillaume Tell de Schiller, juré leur alliance fondatrice de la Confédération. Rolland utilise la version allemande, alors qu'en Suisse romande on appelait le lieu «Grütli».

I5. Trad. : "Ton heure a sonné.» 
nous, on se fiche des gros bonnets de Berlin. - Attendez quand la guerre sera finie... On ne sera plus l'Allemagne de 1914. Si alors nous avions vaincu, tout à fait vaincu, cela aurait été difficile pour le peuple. Mais dans les années de guerre, le peuple s'est dégourdi, réveillé, tous ils voient le monde; tous travaillent et vivent pour un même but... Tenir bon à tout prix, mais pour tous." - Je ne sais pas exactement comment on travaille en France, en Angleterre... Je n'ai vu que le travail de guerre en Allemagne, et je ne pouvais m'empêcher de l'admirer. On a tant écrit, dans les camps opposés, qu'on n'y travaille qu'à coups de fouets et de fusil! ... Je puis dire franchement: "Ceci n'est pas vrai." J'ai vu un dévouement incomparable, simple et spontané. Les dirigeants n'ont qu'à placer, situer les volontés des particuliers. Les enfants et les vieux, les fermmes du peuple surtout, accomplissent des tâches dont on ne les aurait jamais jugés capables. Une grande tristesse intérieure respire dans ce peuple. Je ne puis lui dire: "Vous êtes coupables. Allez! Votre mort me ravit. Je vous hais!» Qui les a vus en 1916 ne peut plus les haïr, sauf les incurables, les "survécus". Il y a une grande espérance pour nous en cette humilité. Ils savent qu'il faut recommencer, qu'il faut tout rebâtir; et leur in sich gehen est sérieux et salutaire. C'est dans le peuple; c'est dans les Universités. Je me rappellerai toujours les paroles courageuses et vraies de $M$. le professeur W. Foerster ${ }^{16}$. Et toute la salle a applaudi, les larmes aux yeux, quand il s'écria:

«Und wir müssen über die heutige Zeit hinausdenken, für ein weitherziges, neues Deutschland das Feld vorbereiten. Es handelt sich nicht darum, für unsere Vorgänger zu leben, die Besten unter ihnen haben für uns gelebt. Gerade weil eingeschränkter Nationalismus nicht wahrheft deutsch ist, bekämpfen wir ihn, mit unserem grössten Goethe. Wenn unsere Jugend nicht vorwärts zieht, sind wir nie des Goethe Wortes würdig:

Was Du ererbt von Deinen Vätern hast

Erwirb es, um es zu besitzen.

Wie verwirklichen unsere Hasser und Schreihälse dieses Wort? Wir werden wieder in Goethe leben müssen, und Menschen sein, bevor wir

I6. Friedrich Wilhelm Foerster (1896-1966): Berlinois, professeur de philosophie et de pédagogie à Zurich, Munich et Vienne, opposant au régime de Guillaume II, hostile à l'emprise de la Prusse sur la culture allemande. Comme pacifiste expatrié, il résidait à Zurich. 
Deutsche sind, denn wenn wir nicht wahrhaft menschlich fühlen und sind, sind wir nicht wert, Deutsche zu heissen. Und auf diesem Boden werde ich immer stehen; keine Gewalt verhindere mich, an einer freien Hochschule, ein wahres Wort zu reden. Ich kann nicht anders und bleibe dabei! ...» ${ }^{17}$

Les applaudissements ne finissaient pas; et beaucoup d'hommes revenus $d u$ front, en uniforme, pleuraient. Un capitaine, en sortant, dit très haut à Foerster: "Ich danke Ihnen. Sie sind ein Mann.» Oh! Il y a eu beaucoup de choses pareilles! C'est comme une marée qui monte... Il est bien possible qu'il faille être très jeune et capable d'illusions pour ne pas défaillir de haine, de découragement et de fatigue. Mais peut-être que l'incapacité de sentir, de mesurer toutes les ruines, tous les efforts mutilés, donne et laisse la force aux jeunes pour bâtir dans les ruines. Je crois la vie éternelle plus forte qu'une guerre pareille. Peut-être nous, nous nous briserons dans l'effort. Mais je crois que dans les grandes souffrances les forces augmentent. J'en ai tant fait l'épreuve! Si j'avais toutes mes forces et ma confiance, je continuerais cette lettre... Mais peut-être en ai-je trop dit, peut-être le zèle m'a emportée trop loin. Je n'accorde point grande importance à mes jugements. Ce ne sont que des choses observées ou des idées acquises seulement après de longues observations. J'ai tâché d'être sincère et équitable, tout en sachant qu'on ne l'est jamais assez. Il faut faire force au cour, et voir, seulement voir. J'ignore si j'ai pu vous blesser comme Français, mais je ne l'aurais jamais pu vouloir. Notre scepticisme suisse nous rend facilement durs et diffciles envers les autres, en apparence. Nous ne serons jamais éloquents: cela nous répugne un peu. Même, j'ai beaucoup de peine à dire à mes amis suisses français des choses qui chez nous ne se disent pas. "Cela se sent." Mais on ne veut pas nous voir avec bienveillance. Chez vous seul, Monsieur, chez M. G. de Reynold ${ }^{18}$ et $M$. Seippel ${ }^{19}$, nous avons pu constater de la compréhension.

I7. Le texte de Foerster d'un cours prononcé à Munich, auquel Elsa Nüesch assista et qui fut perturbé, fut traduit en français dans l'édition du $J A G$ de 1952, p. 1855. Voir aussi l'entretien du 28 mai 1917.

I8. Gonzague de Reynold (1880-1970). En 1916, il n'était alors pas encore le propagateur de l'autoritarisme conservateur des années 1930, mais cofondateur de la Nouvelle Société Helvétique et soucieux de l'entente entre Alémaniques et Romands. Sous l'impulsion de Carl Spitteler, il promouvait alors le rapprochement entre Romands et Alémaniques et la neutralité.

19. Seippel publia l'article "Au-dessus de la mêlée» les 22 et 23 septembre 1914 en tant que rédacteur du Journal de Genève. 
On est des "Landvogts von Greifensee, des Wertmüller " ${ }^{20}$ et on a un seul grand Evangile: aimer notre terre et tous les hommes. C'est presque trop simple de le dire...

Elsa Nüesch

No3: Romain Rolland à Elsa Nüesch, 31 décembre 1916

Dimanche 31 déc[embre] 1916

Chère Mademoiselle

Vous avez écrit une belle et poignante lettre, dont je vous remercie. Comment pouvez-vous craindre qu'il y ait là le moindre mot pour me blesser? Chaque souffrance humaine est mienne, et je suis heureux de tout ce que je vois de grand, où que ce soit. Je vous serai reconnaissant de me communiquer tout ce que vous pouvez encore apprendre d'intéressant.

Je crois que cette cruelle épreuve sera salutaire à plus d'une nation. (Pas à toutes peut-être: il en est dont les sources de vie auront été trop profondément atteintes par la guerre). De toute façon, l'humanité s'en relèvera, et ces grands chocs de peuples se résoudront en de plus vastes groupements, qui s'acheminent lentement à l'unité. Mais l'énigme douloureuse, c'est que tous les progrès s'achètent et que les fautes se payent au prix du sang des millions d'innocents.

Une minorité de femmes françaises ont maintenu leur esprit hors des atteintes des passions nationalistes. M'autorisez-vous à donner votre adresse à certaines d'entre elles, qui souhaiteraient de vous connaître?

Je vous envoie, chère Mademoiselle, mes meilleurs vœux pour votre santé (j'espère qu'elle est à présent rétablie de la maladie qui vous a forcée à interrompre votre lettre). Veuillez croire à mon cordial dévouement

Romain Rolland

20. Personnages historiques et romanesques du XVII e suisses aussi décrits par l'écrivain Gottfried Keller. 


\section{Sierre, Hôtel du Château}

Ci-inclus un article récemment publié par la $N$. Zürcher Zeitung, et qui va paraître en français dans la revue Le Carmel $^{21}$ de Genève. - Vous lirez aussi dans le $\mathrm{n}^{\mathrm{o}}$ de la revue Demain, qui est sous presse, un article plus important qui s'adresse à tous les peuples ${ }^{22}$.

\section{No4: Romain Rolland à Elsa Nüesch, 2 mai 1917}

mercredi 2 mai 1917

\section{Chère Mademoiselle}

Merci de votre lettre, - toujours si pleine de vie, si "savoureuse", dans tous ses jugements, qu'ils soient justes ou non, mais ils sont toujours sincères, et vous vous y mettez tout entière.

Et merci pour l'excellent résumé de mon article dans le St.Galler Tagblatt ${ }^{23}$. Une petite erreur seulement: ce n'est pas d'Allemands dont il est question dans les expéditions de Mandchourie, mais de Russes. La campagne de Chine est tout à fait différente; et là, toutes les grandes puissances ont été associées, sous le commandement accepté d'un généralissime allemand. Ce qui est la part spéciale de l'Allemagne dans le réquisitoire du début, c'est le Damaraland ${ }^{24}$.

2I. Rolland fait allusion à Der steile Pfad (La route en lacets qui monte) qui parut dans la Neue Zürcher Zeitung de Noël, du 24 décembre 1916 (n²125) et dans Le Carmel, Genève, janvier 1917 (article repris dans L'esprit libre). Comme Les Tablettes de Claude LeMaguet (alias de Jean Salives), cette revue pacifiste, publiée par le psychologue Charles Baudouin, faisait partie de l'entourage de Rolland et des pacifistes expatriés résidant à Genève entre 1914 et 1918.

22. Aux peuples assassinés, dans demain, Genève, $\mathrm{n}^{\circ} 11 / 12$ nov.-déc. 1916. Les fascicules de demain paraissaient régulièrement en retard, ce qui explique pourquoi Rolland annonce le 31 décembre 1916 un numéro qui porte la référence nov.-déc. 1916.

23. E.N. (Elsa Nüesch, Neuchâtel im April) : Romain Rolland an die Völker Europas, dans St.Galler Tagblatt, 24 avril 1917, en première page. Nüesch résume la pensée de Rolland, son pacifisme, son universalisme et son appel au renouvellement. Elle rend compte de l'appel Aux Peuples assassinés. Voir aussi le commentaire de Rolland, JAG, p. 1212.

24. Les Damars (ou leur territoire Damaraland, dans la colonie impériale allemande africaine, actuellement en Namibie). Les Damars, comme aussi les Ovambo ont été l'objet d'une campagne d'extermination allemande lors de la soumission des Hereros par le corps expéditionnaire allemand du comte Lothar von Trotha, en 1904. Rolland 
Le temps me manque pour vous écrire plus longuement aujourd'hui; mais je tenais à vous remercier sans tarder.

A vous bien cordialement Romain Rolland

Lisez-vous parfois la petite Revue mensuelle 25 ? J'y ai cité dans le dernier $\mathrm{n}^{\mathrm{o}}$ des textes émouvants de soldats français.

- Vous savez que les amis Jouve sont établis à Genève, 25 rue Merle d'Aubigné, et qu' il y a quelque espoir que la revue "Demain ${ }^{26}$ » renaisse.

\section{No5: Visite d'Elsa Nüesch à Romain Rolland à Villeneuve, 28 mai 1917}

$J A G$, p. 1206 :

28 Mai Visite de $M^{1 l e}$ Elsa Nüesch, avec qui j’ai quelques fois correspondu. Très germanophile au début de la guerre, elle a complètement évolué, depuis. Tout ce qu'elle me raconte de son séjour l'an dernier, en Allemagne, souligne l'extrême misère, les souffrances sans nom des soldats et de la population affamée, et en même temps leur incurable passivité, leur neurasthénie profonde et découragée. Ce qui ressort d'ailleurs de toutes les conversations, c'est l'effrayante disette d'individualités libres dans le monde. Et même la diminution générale de vitalité profonde. On dirait que l'âme humaine fuit, comme un vase fêlé.

critique Nüesch pour avoir confondu les campagnes de von Trotha en Afrique avec celle de Chine, en 1900, (la révolte dite des Boxers) commandée par le comte Alfred von Waldersee, mais qui fut, celle-là, une expédition composée de troupes internationales.

25. La Revue mensuelle était une petite revue de variétés littéraires. Voir Ph. Monnier, «Romain Rolland et la Revue mensuelle de Genève».

26. demain, (titre exact en minuscules) Genève 1916-1918, revue d'aspiration pacifiste, anarcho-socialiste et internationaliste publiée par Henri Guilbeaux, qui servit d'organe de publication aux expatriés pacifistes en Suisse qui entouraient Rolland. En mai 1917, la revue dut interrompre sa publication faute de fonds qui ne furent renfloués qu'en automne de cette année. Interdite en France, la revue postula la reconstitution de l'Internationale socialiste et s'affilia à la révolution bolchevique, en 1918. Pour avoir publié demain, Guilbeaux fut condamné à mort en contumace en France pour «intelligence avec l'ennemi», en février 1919. 


\section{Entretien de Romain Rolland avec Elsa Nüesch du 28 mai 1917 à la villa Olga à Villeneuve}

De cette entrevue est conservé un témoignage de la plume d'Elsa Nüesch pour un cours qu'elle a prononcé d'abord à l'Université populaire de Neuchâtel, en 1926 (à l'occasion du 60e anniversaire de Rolland) et puis à la Handelshochschule St.Gallen (aujourd'hui Université de Saint-Gall) en 1936 (à l'occasion du 70 ${ }^{\mathrm{e}}$ anniversaire de Rolland) ${ }^{27}$.

Au début il m'intimidait. Il n'avait pas du tout l'air des personnages célèbres vaniteux qui aiment parler d'eux. Tout de suite, il voulut tout savoir de moi. Evidemment, la conversation ne cessa de tourner autour de l'énigme de l'Allemagne. J'avais été en Allemagne, y avais étudié, y avais voyagé. Y avait-il une science allemande qui ne se soit pas inféodée à la propagande de guerre? - Je ne pus lui cacher qu'à Munich il n'y avait que peu de personnages, comme par exemple Heinrich Wölfflin ${ }^{28}$, qui nous offrait une vision libre d'un arrière-goût politique de l'art ou des impressionnistes français comme il le fit en temps de paix. Mais déjà von der Leyen, dans son histoire de la littérature, prétendait que Spitteler n'était qu'une "version de poche" de Nietzsche dont il aurait copié les meilleures idées. Le débat autour de Friedrich Wilhelm Foerster, qui avait fustigé la politique de Bismarck et son emprise prussienne dans un article de la Friedenswarte ${ }^{29}$ de Zurich fut houleux. Cet article avait été repris, mais tronqué, par des journaux allemands. Il fut vilipendé de façon haineuse. Lorsque j'entrai, un soir, sans m'en douter, dans la salle de cours, je devins témoin involontaire d'une scène brutale. De tout jeunes étudiants, certainement pas des combattants du front, se

27. Seul le manuscrit de cours (en allemand) est conservé au Gosteli-Archiv (cote 590-1-00). Extraits traduits par mes soins. Les principales phrases-clefs sont citées en français par Elsa Nüesch. Elle y cite la lettre de Rolland du 4 août 1917, traduite, reproduite ici en langue originale. Elle a en outre publié des articles de félicitations dans divers journaux suisses alémaniques lors des $60^{\mathrm{e}}$ et $70^{\mathrm{e}}$ anniversaires.

28. Heinrich Wölfflin (1864-1945) grand historien de l'art suisse, successeur de Jakob Burckhardt à la chaire d'histoire de l'art à Bâle et, subséquemment, à Berlin.

29. Die Friedenswarte, $1915, \mathrm{n}^{\circ} 17$ p. 25 et $165 ; 1916, \mathrm{n}^{\mathrm{o}} 25$ p. $18 ; 1917, \mathrm{n}^{\mathrm{o}} 1$, p. 19. Cette revue fut le principal organe du mouvement pacifiste international, initié par Bertha von Suttner, publiée par l'Autrichien A. H. Fried (1864-1921). Entre 1915 et 1919, elle s'était repliée sur territoire neutre, à Zurich. 
rassemblèrent dans l'arrière de l'auditoire et se mirent à faire du tapage lorsque le professeur se montra. Foerster resta calme, mais un coup de feu éclata subitement qui se planta dans le tableau noir. C'est alors seulement que quatre filles allemandes et nous, trois étudiants suisses, qui intervinrent pour assurer de force la sortie sauve de Foerster. Ce comportement collectif de horde et de meute nous surprit. Nous ne nous étions pas étonnés que l'on fasse du tapage contre des enseignants impopulaires, mais nous étions perplexes de voir, sans comprendre pourquoi, éclater un tumulte au commandement d'une seule voix. Quand on leur parlait individuellement, ils avaient l'air raisonnables, mais quand ils étaient ensemble, leur esprit de corps patriotique exigeait d'eux de vociférer en union, alors que chacun, seul, ne l'aurait osé.

Un moment de silence - Monsieur Rolland souriait étrangement "C'est bien une réaction suisse [en français dans le texte allemand]. D’abord vous mettez longtemps pour comprendre de quoi il s'agit. Alors, votre notion du droit auquel vous êtes sensibilisés, perce. Vous faites spontanément ce qui est juste, mais seulement au dernier moment. Vous protégez l'enseignant menacé et le placez au milieu de vous. Vous faites ce que votre peuple aurait dû faire dès le début de la guerre, sans peur ni hésitation. Vous sous-estimez votre crédit moral auprès des peuples combattants. Vous courez le risque de rater la vraie vie dans votre sommeil. Il faut qu'il y ait une catastrophe, qu'un coup de feu frôle vos oreilles, pour que vous compreniez que vous vous trouvez au milieu de la mêlée et qu'on fait appel à vous. Mais vous préférez vous tapir derrière vos pots de géraniums, contempler votre beau pays, être en harmonie avec le monde pour autant que vous vous portiez bien. Même là où vous vous échauffez, vous le faites comme les gens de Seldwyla ${ }^{30}$. Vous ne risquez jamais votre peau. Vous ne vous consumez pas en passions. C'est ce qui manque à la Suisse. La lutte et les combats dans les grands cercles vous sont étrangers. Votre horizon intellectuel est étriqué. Avec l'âge vous vous isolez et devenez des marginaux. Vous craignez le contact avec le vivant, avec le monde hors de vos frontières. Dans le domaine financier aussi, vous ne risquez pas trop. Pas d'argent, pas de Suisses [en

30. Allusion au recueil de Gottfried Keller Die Leute von Seldwyla: en Suisse l'expression courante d'un provincialisme suffisant et nombriliste: analogue à l'expression allemande du "Schildbürgertum». Rolland était un excellent connaisseur de la littérature suisse alémanique - comme André Gide. 
français dans le texte allemand]. Par ailleurs, vous admirez aveuglément tout ce qui vient du dehors: il suffit d'un commis-voyageur baragouinant pour vous faire entourlouper. Votre presse et même vos professeurs reprennent les mensonges de l'extérieur.»

"Mais il incombe à vous-mêmes de frayer votre chemin, comme l'ont fait des Burckhardt ou des Spitteler. Apprenez à penser avec votre propre tête. Ne pensez pas seulement à vous-mêmes, mais aussi aux peuples autour de vous qui souffrent, qui ont perdu la bonne mesure. Voici ce qui serait la mission exemplaire suisse. Vous êtes trop commerçants et ne voulez pas faire tort à vos clients ici et ailleurs si vous persistez dans votre opinion. Vous faites l'impression de portiers d'hôtel qui s'inclinent devant n'importe quel client et lui donne raison, pour autant qu'il vous paye monnaie comptant.»

Ces propos m'ont irritée, d'autant plus que je ne pouvais me cacher que beaucoup de remarques étaient bien fondées. Néanmoins, je pensais que M. Rolland généralisait un peu les choses. Je ne voulais pas avaler tout. Il fallait que je m'explique.

"Voyez-vous, M. Rolland, les défauts que vous relevez, nous les voyons aussi et ils sont l'objet de débats passionnés chez nous. Partiellement ils proviennent de l'industrie du tourisme, qui rapporte de l'argent au pays, mais empoisonne moralement les populations des montagnes. J'ai honte de tout montagnard qui se prostitue à d'indignes besognes pour un pourboire. Et puis, nos commerçants sont devenus tels, parce qu'ils ont dû se faufiler entre les blocs des grandes puissances. Et il y a en général les anciens. Ce qui se dit dans les journaux, ce n'est pas la voix du peuple, certainement pas la voix de la jeunesse. Ce fut un événement que moi, petit môme de 22 ans, aie pu m'exprimer dans un journal. Les rédacteurs sont à nouveau totalement sous l'emprise des idéaux anciens. Aucun d'entre eux, sauf un tout jeune, n'a voulu me croire quand je disais que l'Allemagne de Weimar est morte, que Beethoven est bien joué, mais que personne ne voit en lui un héros de la liberté. L'Allemagne des romantiques perdure encore dans les hommes d'aujourd'hui, mais, en réalité, elle est perdue. Tous ces vieux messieurs sont encore empêtrés dans les préjugés et les routines d'antan, de l'époque du vieux Heidelberg. Si on leur dit que tout cela est perdu, ils se fâchent et nous ferment la bouche, car nous serions trop jeunes et inexpérimentés. Pourtant, ce sont les jeunes qui payent les fautes des anciens dans les pays en guerre; et s'il y avait des combats chez nous, il en serait de 
même. On nous accuse constamment de manque de respect, mais est-ce nous qui sommes coupables de cette guerre? Est-ce nous qui avons forcé la course aux armements? Est-ce nous qui avons rendu notre industrie si dépendante de l'étranger? La population estudiantine réfléchit, pas seulement les ouvriers. Nos soldats, nos œuvres de la Croix Rouge sont témoins de la misère. Mais nous sommes condamnés au silence. Nous prendrons néanmoins la parole le temps venu.»

"Alors» m'interrompit-il: «que ferez-vous alors?»

"Alors nous voulons d'abord faire l'ordre chez nous [en français dans le texte allemand]. Nous avons de grands projets. Nous voulons davantage de justice sociale, nous voulons nous affranchir de la tutelle économique étrangère. Nous devons surmonter nos conflits internes et, en même temps, réconcilier au niveau intellectuel les peuples. Nous plaçons la notion de la solidarité humaine au-dessus de celle de l'hégémonie. Voyez-vous, si je dis cela dans la confusion, il faudrait que vous rencontriez d'autres personnes, par exemple de notre association estudiantine la Zofingia ${ }^{31}$. Parmi nos camarades il y a des têtes brillantes. Bien sûr que nous sommes tous jeunes et en effervescence, mais nous cherchons honnêtement la clarté. Il y aurait là quelques camarades historiens qui pourraient faciliter bien mieux que moi l'entente entre les peuples.»

Au lieu d'une réponse, il me perça d'un long regard silencieux jusqu'à ce que je me taise. Pourquoi me dévisageait-il ainsi? Qu'ai-je dit de sot?

Alors, lentement et de façon insistante, il se mit à parler et chaque mot eut son poids:

"Vous venez de me dire que vos camarades peuvent faire bien plus que vous. Vous allez prétendre qu'ils valent mieux que vous? Ils semblent vous en imposer, ces camarades ...» (ces paroles étaient accompagnées d'un fin sourire narquois, mais, subitement, il se leva et se planta

3I. "Société de Zofingue» (Zofinger Verein, nommé selon la localité de Zofingen au canton d'Argovie) importante association estudiantine de tradition radicalo-libérale à laquelle a adhéré un nombre important de personnages clefs de la vie politique et culturelle de la Suisse. Contrairement aux associations estudiantines d'inspiration germano-prussienne en Suisse, la Zofingia propageait une société civile démocratique et ouverte. C'est sans doute Elsa Nüesch, proche de cette association, qui a établi le lien entre Romain Rolland et la Zofingia. Le lendemain de l'entretien, le président Julius Schmidhauser écrit à Rolland pour lui témoigner son admiration et son adhésion à son attitude devant la guerre. Voir à ce sujet: $J A G$, p. 1216-1217 et Les Précurseurs, où Rolland a repris un article qu'il avait consacré à la Zofingia. 
devant moi). "Savez-vous bien ce que vous dites?» - Je commençais à me sentir mal à l'aise - «Mais... bien sûr, voyons, ce sont des hommes, ils entreront dans la vie publique, ils vont y jouer un rôle, tandis que moi... "... tandis que vous?"

«... tandis que moi, je ne peux pas faire grand'chose. Une femme est réduite chez nous à un domaine de vie réduit et étroit."

«Ne vous imagineriez-vous pas cela faussement? Votre comparaison n'est-elle pas fausse? - Vous vous comparez à des hommes de la sphère publique, qui ne se salissent pas seulement les mains mais aussi leur conscience dans cette activité publique. Peut-être que vous trahirez un jour vos idéaux de jeunesse pour faire carrière. Dans leur jeunesse ils se donnent des idéaux. Cela ne vaut pas grand'chose, car ils ne portent pas encore le fardeau de la famille. Plus âgés, ils s'inclinent devant le veau d'or. - Une femme a d'autres moyens pour agir, de transmettre le flambeau de la foi à la génération suivante. Restez fidèle à vous-même, mais grandissez. Faites ce que vous pouvez là où vous êtes. Si vous n'accomplissez rien d'autre que ce que dont vous êtes capable, c'est déjà suffisant pour toute une vie. La plupart des hommes et des femmes ne le font pas. Combien rares sont celles et ceux qui peuvent prétendre: J'ai donné toute ma mesure. J'ai servi mon Dieu de toutes mes forces. - La tâche est grande, mais nous ne pouvons achever en un jour que ce que ce dont ce jour est capable. - C'est assez. - La vie entière est une lutte. La vie, voyezvous, n'est autre qu'une victoire durable, comme m’affirme un médecin. La vie est la victoire qui dure [en français dans le texte allemand].»

\section{Commentaire de Romain Rolland à la suite de cet entretien}

$J A G$, p. 1212:

L'autre jour je disais à Elsa Nüesch, qui a réussi à donner des fragments de mon article Aux peuples assassinés, dans un journal de SaintGall: "Tout de même, voyez comme dans toute la Suisse, allemande et romande, on fait le silence sur cet article. "Korrodi ${ }^{32}$, de la Neue Zürcher

32. Eduard Korrodi (1885-1955), journaliste et critique littéraire influent, longtemps chef du feuilleton de la Neue Zürcher Zeitung (NZZ). La NZZ eut une attitude ambivalente à l'égard de Rolland: elle le respecta, mais prit de plus en plus ses distances au fur et à mesure que Rolland prit parti pour la gauche dans les années 20 et 30 . 
[Zeitung] (avec qui je suis en bonnes relations), en a reçu un exemplaire, m'en a accusé réception, m'a promis d'en parler et pas un mot n'en a été dit dans son journal, dont je suis pourtant un collaborateur. Elsa Nüesch me donne le mot de l'énigme. La Neue Zürcher est dans les mains des gros fabricants de munitions pour... pour les deux camps. Et partout, c'est ainsi. Les ouvriers suisses qui travaillent dans les ateliers de munitions sont enragés pour que la guerre continue, avec les bénéfices qu'elle leur apporte ${ }^{33}$.

No6: Romain Rolland à Elsa Nüesch, 5 juin 1917

mardi 5 juin 1917

\section{Chère Mademoiselle}

Nos lettres se sont croisées.

Non, s'il vous plaît, ne parlez pas à la jeunesse suisse (ou de la jeunesse suisse), en mon nom, - mais au vôtre. Ce que j'ai à dire publiquement, je l'écris moi-même. Et ce que je dis à mes amis, c'est pour mes amis seuls. Veuillez expliquer à votre correspondant de Berne qu'il s'est mépris sur ce que vous lui avez écrit de ma pensée. Il ne peut me venir à l'idée de juger un peuple en général, ni surtout une génération nouvelle qui ne s'est pas encore exprimée. Personne n'est plus ennemi que moi des jugements catégoriques, des généralisations insuffisamment vérifiées. Personne ne fait plus crédit à la vie et n'a plus de respect pour ses nuances infinies.

J'ai mes raisons pour demander à mes amis d'être discrets sur ce que je leur confie. Je sais parfaitement (c'est la règle) qu'une pensée intégrale ne peut, si elle est libre, être comprise de tous et doit même, si elle est divulguée sans prudence, amener des malentendus fâcheux, - fâcheux non pour moi, mais pour le public même à qui je ferais du mal en voulant lui faire du bien. La pensée est aussi délicate et dangereuse à manier qu'un explosif moderne: on ne peut la confier à des mains d'enfant. Il

33. L'historiographie a donné raison à Elsa. Les effets économiques et sociaux de la guerre étaient régionalement sélectifs selon les secteurs en Suisse: alors que certaines branches chômaient, d'autres fleurissaient, dont l'industrie des machines fournissant des armements, où les salaires en augmentation compensaient l'inflation galopante des prix. Ailleurs, dans les grandes villes, il y eut - comme en Allemagne - des manifestations violentes de femmes assaillant des magasins d'alimentation. 
faut y aller doucement, avec une sollicitude fraternelle. J'ai souvent envié les Grecs qui avaient deux publics séparés: les initiés et la foule. Le problème de notre époque démocratique est qu'on est obligé d'offrir une nourriture égale à des âmes inégales. Les unes en profitent, les autres en deviennent folles.

Je vous retournerai ces jours-ci, vos livres et brochures. Je connais ces dernières. Quant aux livres, je crains de ne pas pouvoir les lire maintenant. Il faut que je m'absorbe et m'isole dans une tâche qui est urgente.

Bien amicalement à vous

Romain Rolland

No7: Elsa Nüesch à Romain Rolland, $1^{\text {er }}$ août 1917

$J A G$, p. 1268 :

Elsa Nüesch, cette étudiante intelligente de Saint-Gall, dont j’ai cité plusieurs lettres, m'écrit ( $1^{\text {er }}$ août):

... Une hostilité sourde et dangereuse est entre les deux âges... De toutes parts, on crie contre le passé. Si seulement nous n'étions pas rongés par le même mal qui a miné les fils de 1813! - le manque d'énergie, une peur devant l'énorme machine sociale, et une grande envie de jouir. Je dois avouer que des personnalités comme le grand Tolstoï nous sont déjà plus étrangères... La vie de Tolstö̈, grande, douloureuse, et saine vers sa fin, nous est trop complexe... J'avoue que je me détourne avec une certaine impatience des grandes âmes tourmentées. Je viens de terminer la lecture de votre Vie de Michel-Ange, et j'en reste fatiguée et plus prise de pitié que d'admiration... Je préfère un Stanley à un Michel-Ange. Des hommes qui marquent une [sic] apogée de la civilisation sont parfois opprimants, et ils ont des faiblesses qui vous éloignent d'eux...

(Jean-Christophe avait aussi prévu cette génération, et il consolait le petit bossu ${ }^{34}$, mal résigné à l'injustice de ses cadets.)

34. Allusion à Jean-Christophe: la fin de l'Adolescent, passage que Rolland mentionne aussi dans la lettre $\mathrm{N}^{\circ} 10$. Voir note 39. 
No8: Romain Rolland à Elsa Nüesch, 4 août 1917

samedi 4 août 1917

\section{Chère Mademoiselle}

«... Un nouvel ordre naissait. Une génération se levait, désireuse d'agir plus que de comprendre, affamée de bonheur plus que de vérité. Elle voulait vivre, elle voulait s'emparer de la vie, fût-ce au prix ... (disons: d'une demi-vérité) ... Aussi lui était-il désagréable d'entendre les grandes voix tourmentées qui lui rappelaient l'existence de la douleur et des doutes: ces rafales, qui avaient troublé la nuit à peine enfuie, qui continuaient, en dépit de ses dénégations, à menacer l'univers, et qu'elle voulait oublier. Impossible de ne pas entendre; on en était encore trop près. Alors, ces jeunes gens se détournaient avec dépit; et ils criaient à tue-tête, afin de s'assourdir ... - Au contraire, Christophe les regardait avec amitié. Il saluait l'ascension du monde vers le bonheur. Ce qu'il y avait de volontairement étroit dans cette poussée ne l'affectait point ... etc.»

$$
\text { La Nouvelle Journée, p. 104-105 } 35
$$

Mais je crois que vous n'avez pas très bien lu Stanley. Ce n'est guère le type d'un "génie heureux". A part quelques moments de joyeuse expansion physique dans ses premières années d'Amérique et dans ses premières explorations, il a toujours une barre de tristesse au front. Même sa femme en convient, - elle qui avait intérêt (naïvement) à le représenter heureux, tout à fait heureux par elle. Il n'a presque jamais su conserver d'amis.

Je vous retourne les deux petits volumes de Federer ${ }^{36}$ et de Kipling ${ }^{37}$ que j’ai lus avec grand plaisir. Et je vous remercie du dernier article Freie Stimmen ${ }^{38}$, qui était très utile à écrire.

35. Référence à l'édition Ollendorff de La Nouvelle Journée; voir le texte dans JeanChristophe, Albin Michel, 1966, p. 1484.

36. Heinrich Federer (1866-1928): prêtre, écrivain et journaliste suisse. Populaire dans la Suisse rurale et catholique malgré le fait qu'en 1902 il fut condamné pour des actes pédosexuels jamais prouvés.

37. J. Rudyard Kipling (1865-1936) : journaliste et romancier britannique célèbre pour son Livre de la Jungle (1894), prosélyte du volontarisme moral, sportif et impérial ayant inspiré le scoutisme. A cette époque, lire du Kipling, était une profession de volontarisme et de modernisme pour une jeune fille.

38. Revue publiée à Lucerne s'adressant à un public catholique de la Suisse centrale. 
Ne vous tourmentez donc pas tant de l'avenir! C'est bien assez du présent. A chaque jour suffit sa peine. - «Le fardeau qui pèse sur votre génération n'est pas du tout effrayant». Il est magnifique. Je voudrais bien être à votre place. Tant pis pour vous (j'entends pour vos compagnons) s'ils ne voient pas leur bonheur! C'est notre génération qui a pâti pour la vôtre (en Suisse) - (pour celle au-dessous de 16 à 18 ans dans les pays en guerre). Tous les matériaux de reconstruction sont là. Il n'y a plus qu'à bâtir. Si la force vous manque, en Occident, tant pis pour vous! Je ne plains pas les faibles. D'autres forts viendront d'Orient ou d'ailleurs. Il faut que la tâche soit faite.

Quant à la peur des "grands neurasthéniques», est-ce la peur de leur faiblesse, ou la peur de leur force? Ces faibles ont bâti Guerre et Paix et la Chapelle Sixtine. - Il faut faire autrement, il faut être autrement; mais il n'est pas nécessaire, pour affirmer sa force, de nier celle d'Hercule. (Encore un neurasthénique, et qui a mal fini!)

Bonnes vacances, et bien amicalement

R.R.

No9: Elsa Nüesch à Romain Rolland, fin août 1917

$J A G$, p. 1295-1296:

... Elsa Nüesch me parle de la misère croissante dans certains cantons de la Suisse allemande:

... Les enfants pour la plupart sont élevés dans l'indigence; je vois beaucoup de cas de pauvreté et de faim cachés, et il ne se passe pas de jour sans que quelqu'un d'entre nous ne découvre une nouvelle misère. Bien des enfants tombent évanouis de faim dans l'école où enseigne ma sour, et les petits sont incapables d'attention, à cause de leur faiblesse physique. Cela n'est pas ainsi dans cette seule école, la misère est partout. La phtisie sévit dans la jeunesse; et un bon médecin nous dit que les privations que doivent endurer la plupart des enfants de tous les pays constituent un facteur de l'affaiblissement général après la guerre. Surtout la faim, et dans tous les pays, le relâchement moral auprès des enfants (ils n'ont plus de père pour les diriger, et leur mère est loin, au travail), tout cela que j'ai observé de près en Allemagne et en Suisse, sera destructeur. - Dans la Suisse française, on n'a pas idée de la misère qui règne ici. Tout est plus cher ici, le climat est fort rude; on dépend d'une industrie pacifique qui chôme tout à fait; on n'a pas d'étrangers, ni 
de fabriques de munitions. La plupart des gens ne gagnent qu'à peine de quoi viure; et comme peu apprécient le privilège de n'avoir pas la guerre, ils sentent leur vide, le manque de travail, et de joie, et de santé...

No 10: Romain Rolland à Elsa Nüesch, 2 septembre 1917

dimanche 2 sep[tembre] 1917

Chère Mademoiselle

Merci de votre bonne et intéressante lettre. - Il n'y a qu'une raison sérieuse pour qu'il ne puisse y avoir de correspondance suivie entre nous: c'est qu'il m'est matériellement impossible de vous répondre. Vos lettres sont si pleines d'idées et de questions qu'il me faudrait un volume pour $\mathrm{y}$ faire la réplique. Et le temps me manque, les tâches s'accumulent.

Donc, pardonnez-moi de ne vous envoyer que quelques mots. Mais croyez au plaisir que j’ai à vous lire et à ma sincère sympathie.

$\mathrm{Ne}$ vous troublez pas de l'avenir. La sagesse des nations dit: "A chaque jour suffit sa tâche "** Et la vôtre (celle de chacun de nous) n'est pas de réformer l'univers, mais de faire un peu de bien et de combattre un peu de mal, dans notre petit rayon. Nous avons tous besoin d'un oncle Gottfried ${ }^{39}$, qui passe parmi nous pour nous consoler, avec un sourire affectueux, de ne pouvoir tout faire. "Als ich kann» (ce qui est de bien mauvais allemand, - mais comme vous savez, je crois, la devise de Jan van Eyck: «Als ik kan»).

Bien amicalement

Romain Rolland

Lisez le livre de Rappoport sur Jaurès ${ }^{40}$. Pour les livres de classe français, il y a trop longtemps que je me suis éloigné de l'enseignement pour être encore au courant. Madame Jouve saura mieux.

39. Jean-Christophe, t. I l'Adolescent, p. 370.

40. Charles Rappoport, Jean Jaurès, l'homme, le penseur, le socialiste, Paris, L'Emancipatrice, 1915. Rolland s'en est largement servi pour écrire son article "Jaurès" paru dans le Journal de Genève, 2 août 1915. Voir B. Duchatelet, «Romain Rolland et Jean Jaurès». 
[en marge:]

** Non: le mot exact est: «sa peine»

No 11: Elsa Nüesch à Romain Rolland, 7 septembre et 4 octobre 1917

$J A G$, p. 1611-1612:

Le plus faible a toujours tort: Elsa Nüesch, cette étudiante suisse, dont j'ai maintes fois déjà apprécié l'observation perspicace, me dit, en deux lettres du 7 septembre et du 4 octobre, combien les Alliés et particulièrement l'Amérique ont évincé l'Allemagne des sympathies des cantons suisses qui étaient outrageusement germanophiles. Ainsi, à Saint-Gall, "C'est une ville de commerce, qui dépend beaucoup de l'Angleterre (le coton qu'on file est anglais); et l'on se découvre toutes sortes de haines contre les Allemands à présent que leur bonheur décline. Mon père même, qui ne connaît ni la France ni l'Allemagne, donne dans le même ton et déteste "le haïssable concurrent". Tout le monde gage pour le plus fort, l'Amérique. Il y a une grande lâcheté dans le jugement des gens... Je n'avais aucune sympathie pour tel ou tel Allemand. Mais aujourd'hui, mon père voudrait me forcer à dire: "Je déteste les Allemands, et je n'épouserai jamais l'un d'eux..."

(A noter que les mêmes gens, il y a deux ans - d'après ce que m'en écrivait Elsa Nüesch - étaient indignés de la haine des Alliés contre l'Allemagne, et, tout en me sachant gré de mes efforts d'impartialité, me reprochaient à moi-même mon injustice à l'égard des Allemands.)

«Le phénomène n'est pas moins frappant au Tessin. Ces Italiens sont pris d'admiration pour l'américanisme. Ils renient leur nature, pour faire une triste copie des Américains... Ils ont une ambition fiévreuse, qui peut se résumer dans ces paroles d'un jeune ouvrier: "Vedrete, non saremo sempre gli ultimi." Je lui répondis: "Bene - sta a provare!" ce qui le fâcha; et il se mit à faire un panégyrique de l'Italie moderne, à quoi je répondis: "Si l'indipendenza, ma i denari inglesi ed americani, quando li pagate?" - "Che m'importa! per ora ci occorre soltano di non esser gli ultimi." - Qui ne l'est plus? Qui est le premier? bien peu! On croit mesurer le progrès aux fils électriques et aux films de guerre. L'Italie se délivre des prêtres, elle méprise le pape pacifiste "un grullo, sciocco, asinone birbante" et on se défait du reste d'estime pour l'Eglise, pour ne plus respecter que la "volontà, il successo, la forza, il volo vittorioso 
del genio fisico, la rigenerazione fisica, l'indipendenza della donna... e l'inappreziabili virtù americane!" Les petites Italiennes s'américanisent. Tout le monde imite un modèle qui n'existe pas. Et pourtant, sous tout ce vernis reste intact l'âme du pays, endolorie, pas trop énergétique, qui tient à ce qu'elle a, plutôt qu'à acquérir ce qu'elle voit en songe. Je crois que demain ou après-demain, ce peuple pris de fièvre sera las, très las... ${ }^{41}$

\section{No 12: Elsa Nüesch à Romain Rolland, 24 janvier 1926}

St Gall, 9 Wienerbergstrasse, 24.1. $1926^{42}$

\section{Cher Monsieur,}

Ne m'en veuillez pas d'un très long silence. La dernière lettre que j'eus de vous fut à Zurich, lorsque je commençai à faire les préparatifs pour ma thèse sur Nietzsche et que je suivais les cours de Ragaz et de Bleuler ${ }^{43}$. Ensuite, ayant quitté Zurich, j'ai dû interrompre mes études pour remplacer un professeur dans notre école supérieure et pour soigner ma chère mère pendant presque une année car alors elle était gravement malade. Ensuite, j'ai terminé mon travail de thèse, qui ne fut pas accepté, et il a fallu le recommencer en 1922 à Zurich et à Neuchâtel, pour ne le terminer qu'en 1924. Du 15.II 1925 au 20.VII

4I. Rolland cite ce passage entre guillemets dans le $J A G$ p. 1611-1612: les originaux de ces lettres d'Elsa Nüesch ne sont pas conservés, on peut néanmoins déduire du contenu que c'est Elsa qui s'exprime ici. Trad: "Vous verrez, nous ne serons pas toujours les derniers." Je lui répondis: "D’accord - mais cela reste à prouver» ce qui le fâcha; et il se mit à faire un panégyrique de l'Italie moderne, à quoi je répondis: «L'indépendance d'accord, mais quand paieront les Anglais et les Américains?» - "Que m'importe! Pour l'instant il ne s'agit que de ne pas être les derniers.» Qui ne l'est plus? Qui est le premier? bien peu! On croit mesurer le progrès aux fils électriques et aux films de guerre. L'Italie se délivre des prêtres, elle méprise le pape pacifiste "un imbécile, un importun, un malfrat» et on se défait du reste d'estime pour l'Eglise, pour ne plus respecter que la «volonté, le succès, la force, le vol victorieux du génie physique, la régénération corporelle, l'indépendance de la femme... et les inappréciables vertus américaines!» Les petites Italiennes s'américanisent...

42. A noter le long silence de huit ans après la lettre précédente.

43. Leonhard Ragaz (1868-1945): professeur de théologie protestante bâlois socialiste et pacifiste; Eugen Bleuler (1857-1939) : célèbre psychiatre zurichois. 
1925 je fus professeur à l'Ecole nouvelle de Bex, j'y donnais des leçons de latin, d'histoire, et j'eus comme élève spécial Olivier Jouve ${ }^{44}$. A Bex, je tombai malade d'une pneumonie, c'est pourquoi [je dus proroger mon projet de thèse]. En été 1925, je quittai l'école nouvelle de la Pelouse, où j'étais venue faire une expérience, dure et salutaire, et je la quittai sans regret, car elle ne correspond pas de loin à ce que devrait être une école nouvelle. En automne, lorsqu'enfin le livre fut imprimé aux «Presses universitaires de France» à Paris, je soutins ma thèse sur Nietzsche et l'Antiquité, essai sur un idéal de civilisation avec M. C. A. Bernoulli (Bâle) et Arnold Reymond (Lausanne) ${ }^{45}$.

Une fois en possession du précieux titre de docteur ès lettres que je mis tant de temps à acquérir, je fus chargée de donner 1) un cours sur Nietzsche à la Handelshochschule St Gallen (en allemand) 2) des leçons de répétition de latin à des élèves du gymnase qui ont de la peine à suivre les cours réguliers. Je donne encore des leçons particulières, travail modeste et assez ingrat, car ce sont pour la plupart des gens nouveaux riches qui font donner des leçons à des enfants peu ou pas capables d'étudier... et enfin je donnai depuis le 15 II 1926 un cours au Volksbildungsbund, sujet proposé par le Comité Romain Rolland ${ }^{46}$, sur l'homme et l'œuvre. Je fais les cours en français, ils sont très suivis par des messieurs presqu'autant que par des dames.

Et ce modeste cours du vendredi a pris une ampleur que je n'espérais pas. On a dû choisir dès la deuxième leçon le plus grand auditorium que la Gewerbeschule possède. J'ai commencé par me demander s'il fallait accepter ou non. Car, bien entendu, je suis résolue de ne pas donner des souvenirs personnels, je n'en ai pas le droit, mais de vous et juste les choses qu'on peut et qu'on doit dire à un public qui est plein de bonne volonté, mais à qui on ne peut pas tout dire... et pour cause. Cependant, je me suis trompée en bien. Les idées ont cheminé plus ces

44. Olivier Jouve, fils d'un premier mariage de Pierre Jean Jouve qui était de l'entourage de Rolland à Genève entre 1914 et 1918. Jouve renia par la suite tout le pan de sa biographie antérieure à 1925. Son prénom a évidemment été inspiré par l'Olivier du Jean-Christophe.

45. Arnold Reymond (1874-1958), pasteur, professeur de philosophie à l'Université de Neuchâtel (codirecteur de la thèse d'Elsa Nüesch avec C. A. Bernoulli), puis à l'Université de Lausanne.

46. Institution précurseur des universités populaires. On apprend ici l'existence d'un "Comité Romain Rolland» dont on ignore les détails. 
dernières années, même chez nous sous la poigne de la misère (notre industrie meurt), beaucoup sont devenus plus clairvoyants, d'autres en revanche plus fanatiques. Des choses qui scandalisaient, il y a cinq ans, on les accepte comme du pain aujourd'hui, c'est-à-dire pour quelquesuns, pour une partie du peuple, nous n'aurons bientôt plus que deux partis qui comptent, les paysans conservateurs d'un côté et les ouvriers de l'autre. C'est précisément à cause de ce cours que je vous écris aujourd'hui déjà afin de vous demander certains détails précis.

La biographie qu'a faite de vous $\mathrm{M}$. Bonnerot ${ }^{47}$, est-elle exacte au point de vue des faits? Elle me paraît bien documentée quoiqu'un peu trop livresque, si vous permettez que je le dise.

M. Bonnerot pourrait-il peut-être me procurer, si je me référais à vous, quelques-uns des articles les plus violents qu'on a écrits contre vous en France, pendant la guerre?

Comment et où pourrais-je me procurer votre Lettre ouverte ${ }^{48}$ à Barbusse parue dans L'Art libre à Bruxelles?

Ayant à ma disposition, un très bel appareil de projections, je serais très heureux [sic] de clore la série des conférences par quelques projections lumineuses. Si je puis avoir le modèle le plus moderne, on peut y mettre n'importe quelle reproduction, pourvu qu'elle soit claire et nette. Pourriez-vous avoir la grande bonté de m'envoyer quelques photographies qui pourraient intéresser? J'aimerais une jolie reproduction soit de Clamecy, soit d'une ville voisine. On connaît très mal chez nous les provinces françaises. Si ce n'était pas trop demander, je serais très contente de pouvoir donner l'image de votre mère et de vous-même. N'allez pas trouver ces prières ridicules ou indiscrètes, je sais que je n'ai pas mérité votre bonté et voici que depuis trois mois, je diffère d'écrire d'un jour à l'autre, de crainte de vous ennuyer par une pareille demande. Si je le fais, c'est d'abord parce que ce n'est pas totalement indifférent quels traits a un homme qui exerce l'action morale qui est la vôtre. Ensuite je montre l'image de Malwida von Meysenbug, il n'est que juste que celle de votre

47. Jean Bonnerot, Romain Rolland, sa vie, son œuvre, Paris, 1921. Pour apprécier les diverses biographies de Rolland, voir B. Duchatelet " "Voulez-vous me permettre" ".

48. Lettre ouverte à Henri Barbusse, Clarté $\mathrm{n}^{\mathrm{o}}$ 6, $1^{\mathrm{er}}$ février 1922, p. 126 sq., préalablement publiée en janvier 1922 dans L'Art libre à Bruxelles. La lettre répond à un article d'Henri Barbusse, L'autre moitié du devoir. A propos du Rollandisme (Clarté $\mathrm{n}^{\circ} 2-3$ décembre 1921, p. 25-28). Cette polémique eut un grand écho. Rolland les republia dans Quinze ans de combat (1935). 
chère [amie] fasse pendant. Je cherche depuis longtemps une bonne photographie de Tolstoï, pas stylisée, mais simple, sans décor et je ne puis la trouver.

En parlant de vous-même, je suis économe en adjectifs, je vous le certifie. C'est à travers votre ouvre surtout que je vous fais parler. Mais puisque vous avez aussi parlé directement, que vous êtes à la fois admiré et combattu, il me faudra bien essayer de vous caractériser, ne fût-ce que pour répondre aux sottises que l'Alliance française ${ }^{49}$ répand sur votre compte, ici comme ailleurs.

Enfin, j'ai souci de donner dans la faible mesure que je puis une impression de vérité à ce que je dis. On me connaît un peu comme ayant des sympathies pour vous et on attend que je donne des faits et que je puisse répondre de ce que je dis.

Pas que les adversaires me manquent, il y a des gens si excités qu'ils prennent mal des choses toutes ordinaires.

Il y en a d'autres qui veulent me prouver que... depuis la guerre vous seriez "mort et malade" à quoi j'ai répondu qu'il se peut que vous ayez été malade de la grippe comme d'autres mais que vous n'êtes pas mort du tout. Je souhaite de tout cœur que votre santé soit bonne. Et d'autres qui vous relèguent parmi les intellectuels, qui ne savent pas prendre parti (ce sont les mêmes socialistes extrémistes qui ne vous pardonnent pas la séparation d'avec Clarté)... mais d'autres, et ce sont ceux-là qui comptent, trouvent dans votre ouvre une lumière qui nous guide, un acte de foi et l'expression d'une conscience. Et ces derniers sont nombreux relativement. Das Spiel von Tod und Liebe ${ }^{50}$, bien donné même sur notre petit théâtre y a beaucoup contribué.

Si vous avez un conseil à me donner, je l'accepterai avec plaisir. Et encore une fois, je n'ai pas la curiosité de journalistes, biographes, si répandue, je tâche de m'assimiler votre ouvre et de donner de mon mieux. Le Schauspielhaus de Zurich m’a demandé de faire une notice pour Aert ${ }^{51}$. J'aimerais bien le voir jouer, mais en français. Par la traduction, même par la meilleure, une ouvre perd un peu de son parfum.

49. Les Alliances françaises faisaient alors fonction de courroies de transmission officieuses pour la diffusion de la culture française en Suisse alémanique: inutile de préciser que Rolland ne faisait pas partie des auteurs favoris.

50. Le jeu de l'amour et de la mort, publié l'année précédente.

5I. Le Schauspielhaus de Zurich représenta Aert à l'occasion du $60^{\mathrm{e}}$ anniversaire de Rolland, le 29 janvier 1926. 
Je me demande à présent que je vous écris, pourquoi je n'ai pas eu le courage de vous écrire plus vite, au cours de ces dernières années. Mais c'étaient des années bien difficiles et j'avais l'impression que je ne pouvais rien faire pour vous. Aussi avais-je besoin d'achever ma thèse que $M$. Reymond voulait très grande, très fouillée en accumulant les difficultés, ayant traité les œuvres de jeunesse de Nietzsche, il fallait refaire Nietzsche tout entier. Il fallait s'enfermer, se soumettre et faire ce grand travail sous le poids de soucis financiers, en donnant (pour décharger mes parents), des leçons particulières... cela ne m'encourageait pas à sortir de moi-même, mais cela aussi était un apprentissage.

Si j'avais plus de temps à ma disposition, je vous dirais volontiers un mot d'Annette et Sylvie, surtout de l'Eté qui m'a vivement intéressée. Je ne sais pas pourquoi cette lecture m'a fait penser au petit Olivier Jouve... et à sa mère et de qui je ne sais plus rien. Je souhaite qu'Olivier trouve pour le guider une main d'homme, jeune et fraternelle, car il a tous les défauts d'un enfant gâté souffrant qu'une mère bonne et douce a gâté. Le séjour à La Pelouse l'a rendu plus dur... mais cela n'était pas ce qu'il lui fallait.

Pardonnez, cher Monsieur, cette longue lettre. Soyez certain de ma reconnaissance que les années et les expériences ont rendue plus forte encore.

Agréez, Monsieur, mes salutations les meilleures.

Votre bien dévouée Elsa Nuesch

No13: Elsa Nüesch à Romain Rolland, 15 avril 1931

Vevey, le 15 avril $1931^{52}$

Cher Monsieur,

Vous m'excuserez quand j'ai osé sonner à votre porte ce matin et je vous remercie beaucoup de votre aimable mot. Le plus curieux, c'est que je n'avais pas même l'intention de vous voir. Un pêcheur du lac m’a

52. Plus de cinq ans séparent cette lettre de la précédente et on sent son caractère improvisé et émotionnel sous l'emprise de n'avoir pas été reçue par Rolland à la Villa Olga le jour même. N'ayant pas sous les yeux cette lettre de 1926, elle y répète quelques détails autobiographiques déjà mentionnés en 1926. 
montré le chemin et m’a conduite jusque devant la porte. Ainsi j’ai sonné malgré mes scrupules.

Je savais par mon amie Odette Richez ${ }^{53}$ à Genève que vous êtes souffrant depuis longtemps.

Je n'ai plus eu le courage de vous écrire, les causes en sont multiples. J'ai fait plusieurs expériences dont une très pénible. Ma chère sœur, mère d'une jeune enfant, est morte voici 3 ans. Polynevrite après les oreillons. Paralysée et morte au bout de 9 jours. De là, grande consternation dans la famille.

J'avais fini mes études et fait mes essais dans l'enseignement. Une année entière j'ai été à la Pelouse sur Bex où l'un des élèves qui m’a été confié en particulier fut: Olivier Jouve. J'ai vécu près de 8 mois avec lui. Cela a été un élève très difficile à mener et très intéressant à connaître, gâté par sa mère qui, abandonnée, l'a chéri et fut très faible envers lui. J'ai vu de près la correspondance d'Olivier avec son père et sa mère et j'ai vu comment une séparation pareille agit sur un enfant (et les adultes s'imaginent que les enfants ne comprennent rien).

De plus, l'Ecole Nouvelle de Bex était loin d'être ce qu'une école d'avant-garde devait être. J'espère pourtant que cette vie un peu rude avec des camarades anglo-saxons aura fait du bien à Olivier (à côté du mal qui y était aussi).

A St Gall, j'ai enseigné les jeunes filles pendant 3 ans. Ayant les enfants de la bourgeoisie aisée, je n'étais pas contente de leur paresse et mes élèves étaient encore moins contentes de moi que je ne l'étais d'elles. J'ai commis la grande erreur de les faire travailler au lieu de faire semblant de travailler. On a si bien manœuvré pour me faire quitter l'Ecole des jeunes filles et me voici depuis 1 an installée comme seule et première professeur femme au gymnase cantonal [fr. : lycée] fréquenté surtout par des garçons de 13/19 ans. Expérience curieuse et qui jusqu'à maintenant a réussi en ce qui touche le rapport avec mes élèves qui est excellent, mais sans le vouloir, sans le savoir ou le provoquer, par le seul fait de réussir, je me suis fait des ennemis parmi certains collègues et certains messieurs de l'Autorité scolaire qui détestent cette sorte de concurrence de la part d'une femme.

De plus, il y a une chose. Il faut apprendre l'art d'avoir des adversaires, de sourire et de les laisser faire.

53. Odette Richez était la traductrice des œuvres de Stefan Zweig. 
J'espère que j'y parviendrai mais pour une femme c'est plus difficile que pour un homme.

De plus, j'ai eu beaucoup à supporter à cause de mes idées. On s'est méfié de moi, m’a écartée dans la concurrence. On a apprécié le cours fait sur Jean-Christophe, en déplorant mon attitude, mais je n'ai pas cédé et c'est heureux. D'après certaines gens, organisateurs de cours, j'aurais dû louer vos livres et passer sous silence les idées d'avant-garde!

Maintenant on ne m'attaque plus, les vieilles familles se retirent, le parti socialiste qui sait ce qu'il veut gagne du terrain. En 10 ans, les idées que nous autres, notre volée de la guerre, avons entretenues contre nos maîtres, notre entourage, elles vaincront.

Mes élèves avancés jouent dans la classe de français des scènes tirées du Danton, ils grandissent en lisant le Jean-Christophe et Les Thibault (R.-M. Du Gard).

En dépit de la crise économique et peut-être à cause d'elle, un désir de renouveau se fait sentir plus âprement quautrefois et le renouveau se fait. C'est ce que je crois avec certitude, après avoir beaucoup douté.

Peut-être que j'aurais préféré être autre chose que professeur, mais les nécessités matérielles m’ont forcée à faire ce terrible métier. Et pourtant maintenant je ne le regrette plus. C'est qu'il me semble que la jeunesse est plus accessible aux vues larges que les gens aux idées faites et telle que la nature m'a faite je ne sais ni flatter les puissants ni renier mes idées ni plier l'esprit à prôner ce qu'il combat.

En lisant l'Ame enchantée, j'ai été surprise de voir combien peu Annette semblait prendre part moralement à son métier, à quel point elle semble le considérer uniquement comme gagne-pain, comme accessoire à côté de sa vraie vie.

Le rapport d'une professeur d'aujourd'hui est beaucoup plus intime, plus camarade et plus direct qu'autrefois.

Le respect ne va plus de soi. Il faut le conquérir, mais pour y parvenir, il faut tout y mettre de soi-même, [se dire] que cela exige une dépense d'énergie inconsciente et très grande.

Je me demande comment une femme s'en acquitte qui est [confrontée à des circonstances semblables]; et la question du progrès féminin n'est pas encore résolue, de loin.

Mais je ne veux pas discuter. Au contraire. Ce qui m’a poussée inconsciemment peut-être à suivre ce brave paysan jusqu'à votre villa, c'est la gratitude de tout ce que vous avez été pour moi et bien plus que 
pour moi, mais pour toute votre génération qui s'évertue à ne pas perdre, mais encore à transmettre à ceux qui viennent le message de La Nouvelle Journée.

J'espère voir Madame Richez à Genève.

Si votre temps et votre maladie vous permettent de me lancer un mot, adressez-le comme par le passé à St. Gallen, 9 Wienerbergstrasse.

J'aimerais tant retrouver les traces d'Olivier Jouve.

Et maintenant en vous remerciant de votre bonté de vous souvenir encore de moi, permettez-moi qu'avec mes sincères vœux pour votre bien-être, je vous exprime ma respectueuse gratitude.

Elsa Nuesch

No 14: Romain Rolland à Elsa Nüesch, 24 avril 1931

Villeneuve, 24 avril 1931

Chère Mademoiselle

Merci de votre bonne lettre. Et excusez-moi encore de ne vous avoir pas reçue.

Non, ma santé n'est pas, à l'ordinaire, mauvaise depuis quelques années; mais j'ai pris, cet hiver, une broncho-pneumonie assez grave; et bien que je m'en remette à présent, je suis tenu encore à des précautions.

En tout cas, le travail n'en souffre pas trop; et le mois récent de maladie a été la seule interruption, depuis quinze ans. J'ai la tête pleine d'œuvres et de tâches.

- Je suis heureux de savoir que vous occupez bien, aussi, votre vie et que vous avez maintenant une activité intéressante ${ }^{54}$.

Je ne défends pas Annette contre vous. Elle n'est pas professeur, par choix et par nature. (Pas plus que moi je ne l'ai été). Mais cela ne veut pas dire qu'elle et moi nous ne reconnaissions pas la grandeur de la tâche et

54. En 1930, Elsa fut nommée professeur au lycée cantonal de Saint-Gall, ce qui était alors très honorifique pour une femme, d'autant plus qu'elle était proche du parti socialdémocrate, très minoritaire dans ce canton. 
son intérêt pour ceux qui sont doués. - Quant aux expériences pendant la guerre, elles ont été un peu spéciales; Jeanne Galzy 55 ma dit les siennes.

Olivier Jouve est un beau garçon, aujourd'hui grand et fort, grâce à Dieu (et à sa maman!) Et heureusement, il paraît bon et reconnaissant.

Mais c'est la pauvre Andrée Jouve qui a été terriblement malade, les années passées; elle a subi de très graves opérations, d'où l'on ne croyait pas qu'elle réchapperait. Elle s'est rétablie, et mène une vie toujours fatigante, à Paris, où son adresse est 13 square de Port-Royal (XIII).

Veuillez croire, chère Mademoiselle, à mon cordial souvenir ${ }^{56}$.

Romain Rolland

Hélas! si les idées de Jean-Christophe paraissent déjà subversives, que dira-t-on de celles de son auteur, qui lui survit? Il a marché depuis! J'étais en train de revoir, en pensée, le voyage de 1914 à 1930, dans une introduction que je viens d'écrire à l'édition allemande qu'on va enfin publier (chez Rotapfel, à Zurich) de mes deux volumes de guerre: Au-dessus de la Mêlée et Les Précurseurs. (Savez-vous qu'aucun éditeur d'Allemagne n'avait osé, jusqu'à présent, publier Au-dessus de la Mêlée? - Les nationalistes de tous les pays sont d'accord contre tous qui ne le sont pas.) ${ }^{57}$

55. Jeanne Galzy (1883-1977) romancière, biographe française, obtint le Prix Femina pour Les Allongés (1923). Connue pour avoir décrit des liaisons lesbiennes (Jeunes filles en serre chaude, décrit l'Ecole normale supérieure pour filles d'avant 1914).

56. La correspondance se termine avec cette lettre, puisque Rolland parle de «cordial souvenir» et que nous n'en avons pas trouvé d'autres. M. Duchatelet a conservé dans ses archives quelques traces d'une suite tardive, provenant des archives de Marie RomainRolland: durant la Seconde Guerre mondiale, en octobre 1944, Elsa Nüesch semble avoir fait parvenir à Romain Rolland un message d'encouragement et de satisfaction de voir la France libérée. Un membre du service consulaire suisse en France aurait fait part à Rolland qu'une "Mlle Elsa Nüesch, Dr ès lettres, demeurant à Saint-Gall (Suisse)» désirait avoir des nouvelles de lui et de sa famille. Un groupe de femmes avait nourri pendant toute la guerre l'espoir d'attirer Rolland à nouveau en Suisse où la villa Olga à Villeneuve lui était restée réservée. Elsa Nüesch en était peut-être.

57. Le Rotapfel-Verlag (Zurich) d'Emil Roniger voulut publier Der freie Geist. Le mécène et éditeur suisse était l'un des quatre éditeurs des œuvres de Rolland en allemand. Il ne put empêcher que le ministère de l'Intérieur du land de Thuringe (Weimar) ordonnât que le tirage du Freie Geist, déjà imprimé à Rudolfstadt, ne fût détruit. A partir de 1934, les écrits de Rolland ne furent plus publiés qu'en Suisse et en Autriche; dès 1938, ils ne l'étaient plus que dans la Suisse isolée. Dans les notes aux Quinze ans de combat, Rolland fustige et raille son éditeur suisse de s'être plié au diktat allemand. 


\section{BIBLIOGRAPHIE}

\section{Fonds}

Fonds Elsa Nüesch (F590), Gosteli-Archiv, Archives sur l'histoire du mouvement féminin suisse Gosteli Stiftung ${ }^{58}$.

Fonds Romain Rolland, Bibliothèque nationale de France, Département des manuscrits.

Fonds Romain Rolland, Bibliothèque Nationale Suisse, et Archives littéraires suisses.

\section{Sources}

Rolland, Romain, Journal des Années de Guerre 1914-1919 et Journal des Années de Guerre (1949-1944), manuscrits, fonds Romain Rolland (F59), Universitätsbibliothek Basel, Handschriftenabteilung.

Rolland, Romain. Journal des Années de Guerre 1914-1919, Paris, Albin Michel, $1952^{59}$.

Il lui en voulut de son attitude anxieuse devant un rejet allemand. Cette crainte n'était pas tout à fait injustifiée, car Roniger, avec sa petite Rotapfel-Verlag, dépendait du marché allemand et avait pris de gros risques et perdu ses frais d'impression pour le stock d'exemplaires déjà produit (Quinze ans de combat, p. LXXVIII).

58. Altikofenstrasse 186, CH-3048 Worblaufen, www.gosteli-foundation.ch

59. La publication de 1952 contient de nombreuses coupures indiquées par des points. Quelques passages concernant Elsa Nüesch ont été précédés ou suivis de coupures. Une vérification sur l'original n'a pas révélé d'anomalies, pas de coupures concernant Nüesch. Voilà pourquoi ces coupures ne sont pas signalées ici. 


\section{Travaux}

Bon Voisinage. Edmond Privat et Romain Rolland, Neuchâtel, A la Baconnière, 1977 (Cahiers suisses Romain Rolland 1).

Duchatelet, Bernard, Voyage à Moscou: juin-juillet 1935, Paris, Albin Michel, 1992.

—, "Romain Rolland et Jean Jaurès", in Jaurès et les écrivains. Actes du Colloque, Orléans, 1993, Orléans, Centre Charles Péguy, 1994, p. 67-75.

—, Romain Rolland tel qu'en lui-même, Albin Michel, 2002.

—, " "Voulez-vous me permettre.” Romain Rolland et quelques-uns de ses critiques", in Lettre et critique, Brest, Publications du Centre d'Etude des Correspondances et Journaux intimes/CNRS-UBO, 2003, p. 357-380.

Meylan, Jean-Pierre, La "Weltbibliothek» et la Maison des Amis, un projet de Romain Rolland (Villeneuve VD, Calcutta et Bâle, 19221926), Brèves, Association Romain Rolland, 2009 (Etudes Rollandiennes 21).

—, "Romain Rolland au-dessus, mais aussi dans la mêlée», in Romain Rolland, une ceuvre de paix. Actes du colloque de Vézelay, 4-5 octobre 2008, éd. par Bernard Duchatelet, Paris, Publications de la Sorbonne, 2010, p. 73-87.

Monnier, Philippe, "Romain Rolland et la Revue mensuelle de Genève», Etudes de Lettres, 3 (1976), p. 1-13.

Reinhardt, Marc, Aux sources du Jean-Christophe de Romain Rolland, thèse, Lyon, 1979.

Romain Rolland et la Suisse, Catalogue de l'Exposition au Musée d'Art et d'Histoire de Genève, 1966.

Stelling Michaud, Sven, Romain Rolland, Neuchâtel, A la Baconnière, 1969. 